\section{Wirksamkeit und Verträglichkeit von Salmeterol in der Langzeit- therapie bei Patienten mit obstruktiver Atemwegserkrankung}

K. M. Beeh, R. Wiewrodt, A. E. Salem¹, R. Buhl für die SAL-MULTI-Studiengruppe

Schwerpunkt Pneumologie, III. Medizinische Klinik und Poliklinik, Klinikum der Johannes Gutenberg-Universität, Mainz
Zusammenfassung: Hintergrund: Salmeterol ist ein lang wirksames, inhalatives $\beta_{2}$-Sympathomimetikum mit einer über 10-12 Stunden nachweisbaren bronchodilatatorischen Wirkung. Methode: In einer prospektiven, offenen, multizentrischen Studie wurden Wirksamkeit und Verträglichkeit einer Langzeittherapie mit Salmeterol (Dosieraerosol, $2 \times 50 \mu \mathrm{g} / \mathrm{Tag}$ ) über im Mittel 29 Monate (Spanne: 4-1145 Tage) bei $634 \mathrm{~Pa}$ tienten ( $54 \%$ männlich, Alter $45 \pm 15$ Jahre) mit leicht- bis mittelgradiger obstruktiver Atemwegserkrankung geprüft, insbesondere auch im Hinblick auf eine Toleranzentwicklung. Peakflow-Werte, Verbrauch an zusätzlichem Salbutamol und Therapieverträglichkeit waren Zielparameter. Ergebnisse: Bereits in den ersten vier Therapiewochen zeigten sich eine deutliche Verbesserung des morgendlichen Peak flows (384 $\pm 104 \mathrm{l} / \mathrm{min}$ auf $413 \pm 112 \mathrm{l} / \mathrm{min}, \mathrm{p}<0,001$ ) und eine Reduktion des zusätzlichen Salbutamolverbrauchs $(21 \pm 21$ auf $8 \pm 14$ Hübe/Woche tagsüber und $9 \pm 12$ auf 4,5 \pm 9 Hübe/Woche nachts, $p<0,001$ alle Vergleiche). Die Peak-flow-Werte blieben über die gesamte Behandlungszeit stabil und lagen bei Therapieende mit $430 \pm 115 \mathrm{I} / \mathrm{min}$ um $10 \pm 18 \%$ höher als vor Salmeteroltherapie $(p<0,001)$. Die häufigsten unerwünschten Ergebnisse waren Exazerbationen der Grunderkrankung (24\%) und Infektionen (12\%), während typische pharmakologische Nebenwirkungen wie Tremor, Herzklopfen und Tachykardie jeweils bei lediglich $<1 \%$ der Patienten dokumentiert wurden. Schlussfolgerungen: Die Befunde bestätigen die Wirksamkeit und gute Verträglichkeit von Salmeterol auch nach mehr als zweijähriger Anwendung. Klinisch relevante Toleranzphänomene der bronchodilatatorischen Wirkung wurden in der Langzeitanwendung nicht beobachtet. Bei Patienten mit mittelgradiger obstruktiver Atemwegserkrankung bietet Salmeterol eine sinnvolle Erweiterung der therapeutischen Möglichkeiten.

Long-term Efficacy and Safety of Inhaled Salmeterol in Patients with Chronic Obstructive Airway Diseases: Background: Salmeterol is a long-acting inhaled beta ${ }_{2}$-agonist with a bronchodilating effect lasting over 10 to 12 hours. Methods: A prospective, open, multi-centre study was performed to evaluate the efficacy and safety of inhaled salmeterol (50 $\mu \mathrm{g}$ BID) over a mean treatment period of 29 months (range: 4-1145 days) in 634 patients ( $54 \%$ male, age $45 \pm 15$ years) with mild to moderate asthma or chronic obstructive pulmonary disease (COPD). Peak expiratory flow rates, rescue use of short acting

Pneumologie 2000; 54: 225-231

(c) Georg Thieme Verlag Stuttgart · New York

ISSN 0934-8387 beta $a_{2}$-agonists and safety were study objectives. Patients were critically monitored for a possible loss of bronchodilator efficacy of salmeterol during long-term treatment. Results: During the first month of salmeterol therapy, morning peak flow improved from $384 \pm 104 \mathrm{l} / \mathrm{min}$ to $413 \pm 112 \mathrm{l} / \mathrm{min}$ ( $p<0.001)$, and use of rescue salbutamol was significantly reduced $(21 \pm 21$ to $8 \pm 14$ puffs/week during daytime and $9 \pm 12$ to $4.5 \pm 9$ puffs/ week during nighttime, $\mathrm{p}<0.001$ both comparisons). Peak flow

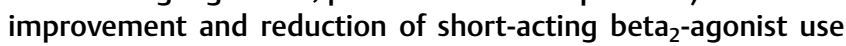
was maintained during the whole study period. Frequent adverse events were exacerbations of the underlying airway disease $(24 \%)$ and infections ( $12 \%)$, while typical pharmacological side-effects like tremor or tachycardia where reported in less than $1 \%$ of all patients. Conclusions: These results confirm the persistent efficacy and favourable safety profile of salmeterol during long-term therapy over more than two years. No clinical signs of a decreasing bronchodilator potency indicating tachyphylaxis were observed. Salmeterol treatment provides a therapeutic option to further improve the management and care of patients with moderate obstructive airway diseases.

\section{Einleitung}

Asthma bronchiale ist eine Atemwegserkrankung mit bronchialer Hyperreagibilität und variabler, reversibler Atemwegsobstruktion, der eine chronische Entzündung der Bronchialschleimhaut zugrunde liegt. Die frühzeitige und regelmäßige antiinflammatorische Behandlung mit inhalativen Kortikosteroiden steht daher im Mittelpunkt der Therapieempfehlungen. Die antientzündliche Therapie wird bei Bedarf durch ein inhalatives kurz wirksames $\beta_{2}$-Sympathomimetikum zur Kontrolle akuter Symptome ergänzt. Sind die asthmatischen Beschwerden trotz einer konsequenten Therapie mit topischen Kortikoiden nicht zu kontrollieren, so sollte die Behandlung um ein lang wirksames Bronchospasmolytikum erweitert werden [1]. Mehrere Untersuchungen bestätigen, dass bei mangelhafter Asthma-Kontrolle die zusätzliche Gabe lang wirksamer inhalativer $\beta_{2}$-Sympathomimetika einer Erhöhung der Dosis topischer Steroide hinsichtlich der klinischen Wirksamkeit überlegen ist [2-4]. Gegenüber einer Dauertherapie mit kurz wirksamen $\beta_{2}$-Sympathomimetika verbessern lang wirksame $\beta_{2}$-Sympathomimetika die Lungen-

${ }^{1}$ Stipendiat des Deutschen Akademischen Austauschdienstes 
funktion, klinische Symptomatik, Nachtruhe und Lebensqualität von Patienten mit Asthma bronchiale bei vergleichbarer Verträglichkeit [5-7]. Im Gegensatz zum Asthma bronchiale ist der Stellenwert der Therapie mit lang wirksamen $\beta_{2^{-}}$ Sympathomimetika bei chronisch-obstruktiver Atemwegserkrankung (COPD) noch nicht in gleicher Weise etabliert, wenngleich eine Reihe von Untersuchungen einen günstigen Effekt auf das Beschwerdebild der Patienten bestätigt [8 - 10].

Aktuelle Untersuchungen deuten nun darauf hin, dass es in der Dauertherapie mit lang wirksamen $\beta_{2}$-Sympathomimetika zu einer Abschwächung zumindest der bronchoprotektiven Wirkung kommt [11-13]. Es ist noch unklar, ob Toleranzphänomene auch die bronchodilatatorische Wirkung betreffen. Die Mehrzahl der bisherigen klinischen Prüfungen mit lang wirksamen $\beta_{2}$-Sympathomimetika wurde über mehrere Wochen bis maximal 12 Monate [14-15] durchgeführt. Beobachtungen über einen längeren Zeitraum lagen bei Studienbeginn nicht vor. Ziel der vorliegenden Untersuchung war es deshalb, bei Patienten mit obstruktiven Atemwegserkrankungen prospektiv die Wirksamkeit und Verträglichkeit einer Langzeitanwendung eines lang wirksamen $\beta_{2}$-Sympathomimetikums (Salmeterol) unter Praxisbedingungen zu untersuchen.

\section{Material und Methoden}

\section{Patienten}

634 Patienten (54\% m, 46\% w; mittleres Alter [ \pm SD] $45 \pm 15$ Jahre, Spanne: 16 - 74 Jahre) mit seit $9 \pm 9,1$ Jahren (Spanne: 0 - 56 Jahre) bestehender leichter bis mittelschwerer Atemwegsobstruktion (Peak-flow-Werte zwischen 50 und 90\% des EGKS-Sollwertes) wurden in 188 Prüfzentren eingeschlossen (3,4 Patienten pro Prüfzentrum). 29\% der Patienten wiesen eine leichtgradige (Peak flow $>80 \%$ ), $71 \%$ eine mittelschwere obstruktive Ventilationsstörung (Peak flow 50 - 80\%) auf. Bei 64\% der Patienten wurde die Erkrankung vom behandelnden Arzt als Asthma bronchiale (ICD-9 493), bei $27 \%$ als chronisch-obstruktive Atemwegserkrankung (COPD; ICD-9 491.2) und bei $9 \%$ als gemischtförmiges Krankheitsbild ohne klare Zuordnung eingestuft.

Es wurde keine strenge Trennung zwischen Patienten mit asthmatisch oder bronchitisch-obstruktiv dominierter $\mathrm{Er}$ krankung angestrebt, sondern lediglich die Indikation zur Therapie mit einem lang wirksamen $\beta_{2}$-Sympathomimetikum gefordert. Die medikamentöse Therapie der obstruktiven Lungenerkrankung und etwaiger Begleiterkrankungen musste in den letzten 4 Wochen vor Studienbeginn unverändert geblieben sein, insbesondere durften die Studienteilnehmer in diesem Zeitraum keine lang wirksamen $\beta_{2}$-Sympathomimetika erhalten haben.

Häufige Begleiterkrankungen waren eine essentielle Hypertonie (30\%), Lipidstoffwechselstörungen (12\%), chronische ischämische Herzerkrankungen (10\%), degenerative Erkrankungen des Bewegungsapparates (7\%) und ein Diabetes mellitus (6\%). Atopische Begleiterkrankungen waren nur bei wenigen Studienteilnehmern bekannt bzw. diagnostiziert worden (allergische Rhinitis: 4\%, chronische Sinusitis: 4\%, atopische Dermatitis: $1 \%$ ).
Ausschlusskriterien waren eine Exazerbation der Atemwegserkrankung und/oder eine Infektion der Atemwege innerhalb von 2 Wochen vor Studienbeginn, eine stationäre Behandlung der obstruktiven Lungenerkrankung in den letzten 4 Wochen vor Studienbeginn, Schwangerschaft bzw. Stillzeit, fehlende Kontrazeption, die Einnahme bronchokonstriktorisch wirkender Medikamente, Alkohol- oder Drogenabusus, eine nach Urteil des Prüfarztes mit der Teilnahme an der Prüfung unvereinbare schwerwiegende Grund- oder Begleiterkrankung sowie die Teilnahme an einer anderen klinischen Prüfung während 4 Wochen vor Studienbeginn. Alle Studienteilnehmer erklärten nach eingehender mündlicher und schriftlicher Aufklärung über Zweck und Durchführung der Medikamentenprüfung schriftlich ihr Einverständnis.

\section{Studiendesign}

Die prospektive, offene, multizentrische klinische Prüfung wurde an 188 Prüfzentren in der Bundesrepublik Deutschland zwischen November 1992 und April 1996 durchgeführt. Prüfzentren waren Praxen niedergelassener Fachärzte für Pneumologie, Innere Medizin oder Allgemeinmedizin. Die Initiierung, Durchführung und Auswertung der vorliegenden Prüfung erfolgte durch die Firma Glaxo Wellcome GmbH, Bad Oldesloe.

Die Prüfmedikation bestand aus Salmeterol-Dosieraerosol (25 $\mu \mathrm{g} / \mathrm{Hub}$, Tagesdosis $2 \times 50 \mu \mathrm{g}$; Glaxo Wellcome GmbH, Bad Oldesloe) und wurde morgens und abends im Abstand von 12 Stunden inhaliert. Zur Kontrolle akuter obstruktiver Beschwerden erhielten die Patienten ein kurz wirksames $\beta_{2^{-}}$ Sympathomimetikum (Salbutamol-Dosieraerosol, $100 \mu \mathrm{g} /$ Hub; Glaxo Wellcome GmbH, Bad Oldesloe). Die bestehende Medikation der obstruktiven Atemwegserkrankung wurde unverändert fortgesetzt, sofern nicht folgende Substanzen eingenommen bzw. innerhalb von 4 Wochen vor Studienbeginn neu angesetzt wurden: orale oder parenterale Kortikoide, inhalative Kortikoide in einer Dosis von $>2 \mathrm{mg}$ pro Tag, Theophyllin, Anticholinergika und andere lang oder kurz wirksame $\beta_{2}$-Sympathomimetika.

Die klinische Prüfung verlief in drei Phasen. In einer vierwöchigen Vorphase erfolgten die Eingangsuntersuchung und die Ausgabe der Prüf- und Bedarfsmedikation. In Phase I der Prüfung wurden die Patienten für 16 Wochen mit Salmeterol behandelt. Am Ende dieser Phase wurde den Patienten angeboten, die Salmeterol-Therapie im Rahmen einer Erweiterung der Studie fortzuführen, da Salmeterol zum damaligen Zeitpunkt in Deutschland noch nicht zugelassen war. Stimmten die Patienten einer Verlängerung zu, wurde in Phase II der klinischen Prüfung die Salmeterol-Therapie für zunächst 6 Monate, später noch zweimal für jeweils 12 Monate fortgeführt, so dass eine maximale Behandlungsdauer von 34 Monaten resultieren konnte. In Phase II (ab Monat 4) konnte die Studie regulär beendet werden, ohne dass dies einem Studienabbruch im Sinne des Protokolles entsprach. Insgesamt wurden pro Patient bis zu 16 Kontrolluntersuchungen durchgeführt.

Prüfplan und Patienteninformationen wurden von der Ethikkommission der Ärztekammer Schleswig-Holstein geprüft und genehmigt. Zusätzlich lagen positive Voten der zuständigen Ethikkommissionen für alle beteiligten Prüfzentren vor. 
Die Studie wurde unter Beachtung aktueller Richtlinien (Arzneimittelgesetz, Good Clinical Practice, Deklaration von Helsinki) durchgeführt.

\section{Lungenfunktionsprüfungen}

Primäres Zielkritikum für die Wirksamkeit der Prüfmedikation war die Veränderung des exspiratorischen Spitzenflusses (Peak flow), der sowohl während des Arztbesuchs als auch in Phase I vom Patienten selbst nach gründlicher Einweisung zweimal täglich (morgens und abends) mit einem Peak-flowMeter gemessen und protokolliert wurde. Die Peak-flowWerte wurden als beste von drei aufeinander folgenden Messungen nach den Richtlinien der European Respiratory Society [16] bestimmt (mini-Wright Peak-flow-Meter, Clement Clarke International Ltd., London, England). Vor den Messungen wurden keine bronchodilatatorisch wirksamen Medikamente appliziert.

\section{Symptomkontrolle}

Sekundäres Zielkriterium war die vom Patienten subjektiv empfundene Symptomkontrolle, gemessen am Bedarf an inhalativen kurz wirksamen $\beta_{2}$-Sympathomimetika, der über ein von den Patienten geführtes Tagebuch erfasst wurde.

\section{Verträglichkeit}

Während des gesamten Prüfzeitraumes wurden vom Prüfarzt unerwünschte Ereignisse (UE) und Veränderungen der Medikation prospektiv erfasst. Vor und nach Phase I der Prüfung wurden zudem Labordaten (klinische Chemie, Hämatologie) bestimmt.

Jedes im Studienzeitraum aufgetretene unerwünschte Ereignis wurde unabhängig von einem möglichen Zusammenhang mit der Prüfmedikation dokumentiert. Unerwünschte Ereignisse waren alle beim Patienten auftretenden Befindlichkeitsstörungen bzw. subjektiven oder objektiven Krankheitssymptome. Als schwerwiegend wurden unerwünschte Ereignisse eingestuft, wenn sie lebensbedrohlich waren bzw. zu einem stationären Krankenhausaufenthalt, einer dauernden und/ oder schweren Behinderung führten, sowie jeder Todesfall und jede maligne Erkrankung.

\section{Statistische Analyse}

Alle Resultate sind, soweit nicht anders angeführt, als arithmetische Mittelwerte \pm Standardabweichung (SD) angegeben. Gruppenvergleiche bzw. statistische Vergleiche klinischer oder funktioneller Parameter vor und unter Studienmedikation wurden mittels t-Test, Wilcox-Mann-Whitney- oder Kruskall-Wallis-Test vorgenommen. Es wurde dabei das Intention-to-treat-Prinzip zugrunde gelegt, d.h. jeder Patient, der mindestens einmal Salmeterol angewendet hatte, wurde in die Analyse einbezogen $(n=634)$. Zur Darstellung zeitabhängiger Veränderungen (Peak-flow-Werte) wurde eine Life-Table-Analyse benutzt. Als stabile PEFR-Verbesserung wurde eine Zunahme von mindestens 201/min im Vergleich zum Ausgangswert definiert, die im weiteren Studienverlauf nicht mehr unterschritten werden durfte. Zusätzlich wurde eine Per-Protocol-Auswertung derjenigen Patienten, bei denen keine Protokollverletzung stattgefunden hatte, durch- geführt $(n=464)$. Fehlende Werte von Zwischenkontrollen wurden nach dem Prinzip „Last Value Carried Forward“ ergänzt. Das Signifikanzniveau wurde bei einer Irrtumswahrscheinlichkeit von $\leq 5 \%$ angesetzt.

\section{Ergebnisse}

\section{Patienten}

634 Patienten wurden für die Teilnahme an der Prüfung rekrutiert. 577 Patienten beendeten die Phase I und 345 Patienten beendeten die Phase II. Dies entsprach einer mittleren Anwendungsdauer von Salmeterol von 29 Monaten (Spanne 4 - 1145 Tage) bzw. 1073 Patientenjahren.

Ein Studienabbruch erfolgte in insgesamt 122 Fällen (19,2\%). Anlässe hierfür (Mehrfachnennungen möglich) waren entweder ein unerwünschtes Ereignis $(n=52)$, eine Exazerbation der Grunderkrankung $(n=21)$, Therapieversagen $(n=6)$ und sonstige Gründe $(n=75)$.

\section{Lungenfunktionsparameter}

Der morgendliche Peak flow vor Therapiebeginn betrug $384 \pm 104 \mathrm{l} / \mathrm{min}$ ( $81 \pm 17 \%$ des Sollwertes). Bereits in der ersten Therapiewoche mit Salmeterol trat eine rasche Verbesserung des Peak flow ein (Patientenmessung, Abb.1). Innerhalb der ersten 4 Wochen nahm der Peak flow auf $413 \pm 112$ 1/min

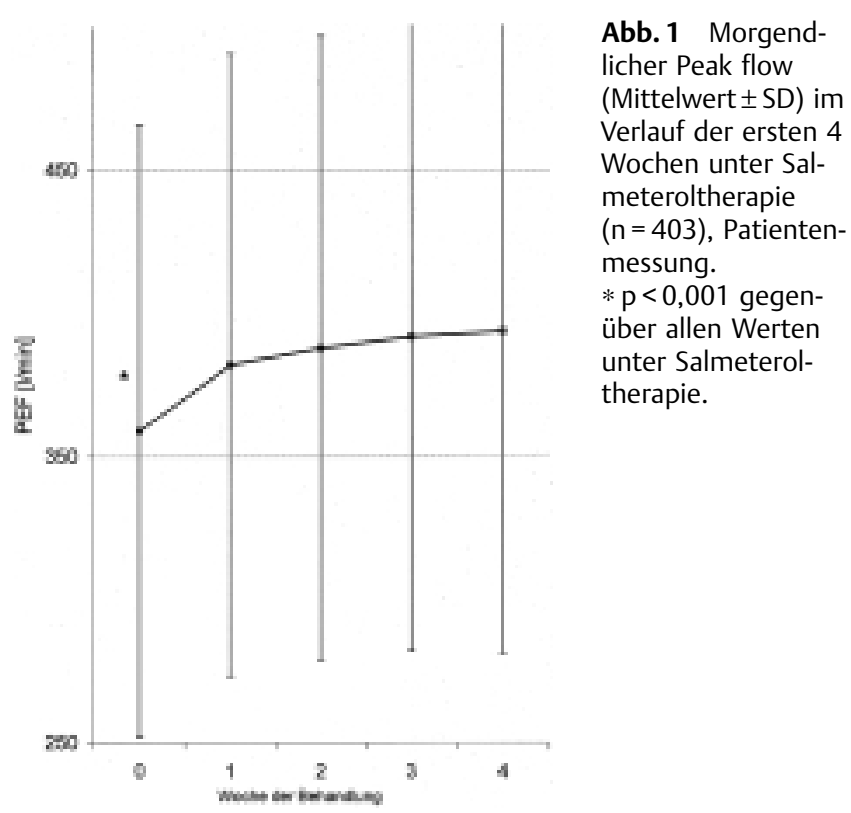

Abb. 1 Morgendlicher Peak flow Wochen unter Salmeteroltherapie $(n=403)$, Patientenmessung. $* \mathrm{p}<0,001$ gegenüber allen Werten unter Salmeteroltherapie.

$(87 \pm 18 \%, p<0,0001)$ zu. Im weiteren Verlauf hielt dieser günstige Effekt an, am Ende der Phase I (Woche 16) hatte sich der Peak flow im Vergleich zum Studienbeginn um $39 \pm 691 /$ min auf zuletzt $423 \pm 1131 / \mathrm{min}$ verbessert $(89 \pm 19 \%$, $\mathrm{p}<0,001)$.

Die signifikante Verbesserung der Lungenfunktion unter Therapie mit Salmeterol bestätigte sich auch im Langzeitverlauf in Phase II (Abb. 2). Wenngleich die Steigerung des Peak 


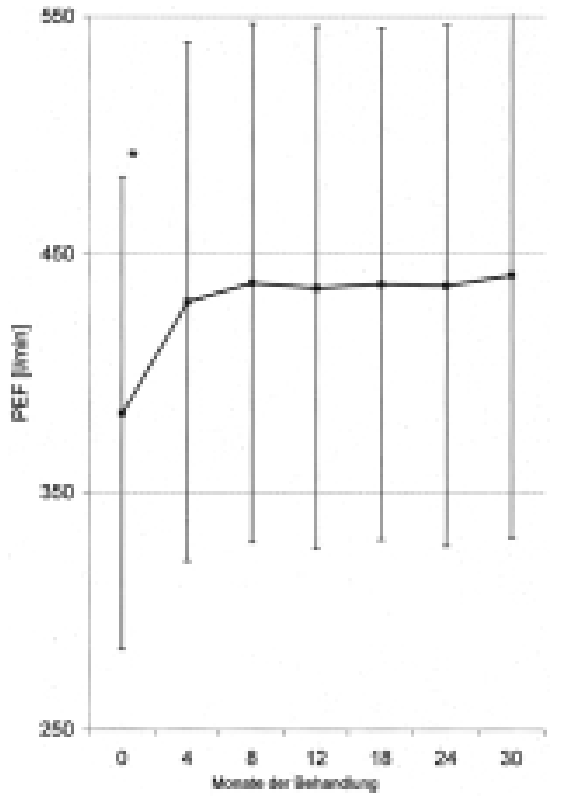

Fig. 2 Peak flow (Mittelwert \pm SD) im Langzeitverlauf unter Salmeteroltherapie $(n=345)$, Arztmessung. $* \mathrm{p}<0,001$ gegenüber allen Werten unter Salmeteroltherapie. flow durch Salmeterol in den ersten Therapiewochen am größten war, kam es auch in den folgenden Wochen und Monaten zu keinem Nachlassen des Therapieeffektes. Selbst in der Plateauphase blieben die Peak-flow-Werte mit zuletzt $430 \pm 115$ l/min ( $91 \pm 20 \%$ des Sollwertes) konstant. Im Vergleich zu den Werten vor Studienbeginn war damit am Ende der Prüfung insgesamt eine Verbesserung um $46 \pm 83 \mathrm{l} / \mathrm{min}$ eingetreten $(10 \pm 18 \%, \mathrm{p}<0,001)$. Dies galt auch, wenn nur die 345 Patienten berücksichtigt wurden, die beide Therapiephasen beendeten (prä: $383 \pm 100 \mathrm{l} / \mathrm{min}$, post: $444 \pm 111 \mathrm{l} / \mathrm{min}$, $\mathrm{p}<0,0001$ ), d.h. die Reduktion des Stichprobenumfangs im Verlauf der klinischen Prüfung war ohne Einfluss auf die Grundaussage der Studie.

\section{Life-Table-Analyse}

$50 \%$ der Patienten hatten nach durchschnittlich 7,4 Monaten (95\% Konfidenzintervall: 5,6 bis 13,9 Monate) eine stabile Verbesserung ihres individuellen Peak flow um mindestens 20 1/min gegenüber dem Ausgangswert erreicht, die im weiteren Studienverlauf nicht mehr unterschritten wurde. Dies galt nach 32,5 (31,6 - 33,8) Monaten für bereits 75\% aller Patienten. Lagen keine Protokollverletzungen vor, vergingen nur 4,4 Monate (3,3-7,2 Monate) bis zum Erreichen einer stabilen Verbesserung des individuellen Peak flows. Eine stabile $\mathrm{Zu}-$ nahme um mindestens 5\% des Solls wurde nach 13,8 (8,421,8) Monaten von 50\% der Patienten erzielt, ein stabil normaler Peak-flow-Wert ( $\geq 90 \%$ des Solls) nach 30,2 (25,2 32,6) Monaten.

Das Ausmaß der Verbesserung der Lungenfunktion war bei Patienten mit Asthma oder COPD nicht signifikant verschieden, es zeigten sich lediglich statistisch nicht signifikante Trends zu besseren Therapieeffekten bei über 60 -jährigen Patienten, bei Frauen und bei Patienten mit einer Erkrankungsdauer unter 6 Jahren.

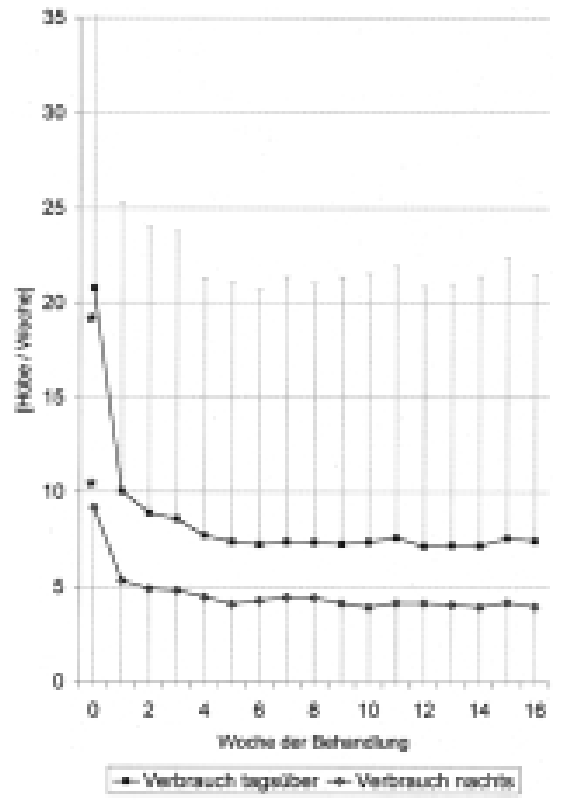

Fig. 3 Wöchentlicher Salbutamolverbrauch (Mittelwert \pm SD) im Verlauf der ersten 4 Monate unter Salmeteroltherapie $(n=403)$. $* \mathrm{p}<0,001$ gegenüber allen Werten unter Salmeteroltherapie.

\section{Symptomkontrolle}

Parallel zur Verbesserung des Peak flows nahm der von den Patienten dokumentierte Verbrauch an bedarfsadaptiertem, kurz wirksamen $\beta_{2}$-Sympathomimetikum zur Kontrolle akuter obstruktiver Symptome und Belastungsdyspnoe unmittelbar mit dem Beginn der Salmeterol-Therapie ab (Abb.3). Bereits innerhalb der ersten Therapiewoche nahm der Salbutamol-Bedarf um 52\% tagsüber und um $42 \%$ nachts ab ( $p<0,001$, beide Vergleiche). Im Therapieverlauf kam es $\mathrm{zu}$ einer weiteren Reduktion, so dass der mittlere wöchentliche Salbutamol-Bedarf am Ende von Phase I (Monat 4) im Vergleich zu den Ausgangswerten signifikant vermindert war ( $-64 \%$ tagsüber und $-57 \%$ nachts; $p<0,001$, beide Vergleiche).

\section{Verträglichkeit}

Die Zahl unerwünschter Ereignisse war in Anbetracht des langen Prüfungszeitraumes naturgemäß groß. Insgesamt berichteten 251 Patienten (39,6\%) über unerwünschte Ereignisse (UE), wobei es sich in 216 Fällen (86\%) um nicht schwerwiegende UE handelte. Angesichts der Grunderkrankungen standen Beschwerden im Bereich der Atemwege mit $34,2 \%$ im Vordergrund, insbesondere Asthma (9,2\%), Bronchitis (7,9\%), Husten $(3,8 \%)$ und Dyspnoe (3,2\%). Häufige andere nicht schwerwiegende UE betrafen gastrointestinale Symptome $(4,6 \%)$, unspezifische Befindlichkeitsstörungen $(8,2 \%)$ und Infekte $(5,7 \%)$.

Bei 40 der 251 Patienten mit UE (6,3\% des Gesamtkollektives) wurde vom behandelnden Arzt ein Zusammenhang des Ereignisses mit der Studienmedikation als „möglich“, „wahrscheinlich“ oder „fast sicher“ angenommen (Tab.1). Diese UE betrafen in erster Linie Symptome der Grunderkrankung (asthmatische Beschwerden, Husten, Dyspnoe) und die bekannten Nebenwirkungen von $\beta_{2}$-Sympathomimetika (Kopfschmerzen, innere Unruhe, Tremor oder Tachykardie) und waren auch häufige Gründe für eine Beendigung der Therapie 
Tab. 1 Sicherheit von Salmeterol während der 34-monatigen Studie: Unerwünschte Ereignisse mit nach Einschätzung des Prüfarztes möglichem, wahrscheinlichem oder sicherem Zusammenhang mit der Prüfmedikation und Anzahl der Studienabbrüche wegen eines unerwünschten Ereignisses insgesamt (UE)

\begin{tabular}{lll}
\hline $\begin{array}{l}\text { Unerwünschtes } \\
\text { Ereignis }\end{array}$ & $\begin{array}{l}\text { Zusammenhang } \\
\text { mit Prüfmedikation } \\
\text { in n Fällen }\end{array}$ & $\begin{array}{l}\text { Abbruch der Studie } \\
\text { wg. UE } \\
\text { in n Fällen }\end{array}$ \\
\hline Husten & 6 & 4 \\
Kopfschmerzen & 5 & 2 \\
Innere Unruhe & 5 & 3 \\
Tremor & 4 & 3 \\
Tachykardie & 4 & 2 \\
Herzklopfen & 4 & 3 \\
Dyspnoe & 4 & 3 \\
Asthma & 3 & 13 \\
schwere Infektionen & 3 & 3 \\
Juckreiz & 3 & 1 \\
Schwindel & 2 & 1 \\
Heiserkeit & 2 & 1 \\
Schlafstörungen & 5 & 2 \\
\hline
\end{tabular}

mit Salmeterol. Seltene als Verdachtsfälle unerwünschter Arzneimittelwirkungen klassifizierte Ereignisse (A: Abbruch der Studie) waren Benommenheit, Arthralgien, Myalgien (A), Gangunsicherheit, Wadenkrämpfe, Konjunktivitis, Geschmacksstörung (A), Glossitis, Übelkeit, Mundbrennen, Gynäkomastie, ventrikuläre Extrasystolen (A), Vorhofflimmern (A), Rhinitis, Soor (je eine Nennung) und Infektionen (A: zwei Nennungen).

In 29 Fällen (4,6\%) wurden die UE als schwerwiegend eingestuft. Diese betrafen operative Eingriffe $(n=6)$, Tumorerkrankungen $(n=4)$, zerebrovaskuläre Erkrankungen $(n=3)$, Hospitalisation aufgrund schwerer Infektionen $(n=3)$, koronare Herzkrankheit $(n=2)$ und periphere arterielle Durchblutungsstörungen $(n=1)$. Arzneimittelnebenwirkungen wie Tremor, innere Unruhe oder Tachykardie wurden lediglich je einmal als schwerwiegendes UE angegeben.

Im Prüfzeitraum traten vier Todesfälle auf, wobei in keinem Fall vom Prüfarzt ein Zusammenhang mit der Studienmedikation angenommen wurde. Ein 44-jähriger Patient verstarb an einem nach Studienbeginn diagnostizierten Rektumkarzinom, ein 42-jähriger Patient verstarb 148 Tage nach Studienbeginn an einem akuten Asthmaanfall, eine 70-jährige Patientin verstarb 17 Monate nach Studienbeginn an einem apoplektischen Insult und ein 70-jähriger Patient erlag 15 Monate nach Studienbeginn einem metastasierten Prostatakarzinom.

Die während der Studie dokumentierten Werte von Herzfrequenz und arteriellem Blutdruck zeigten im Verlauf keine signifikanten Veränderungen, insbesondere keinen Trend zu Tachykardie oder Hypertension. Die Laborwerte (Hämatologie, klinische Chemie) blieben im Mittel im Normbereich, es traten keine klinisch relevanten Veränderungen der Laborwerte auf, die auf die Studienmedikation zurückzuführen waren.

\section{Diskussion}

Die Ergebnisse dieser klinischen Prüfung bestätigen, dass lang wirksame $\beta_{2}$-Sympathomimetika bei Patienten mit obstruktiven Atemwegserkrankungen auch in der Langzeittherapie unter Praxisbedingungen wirksam und gut verträglich sind.

Unter Therapie mit Salmeterol $(2 \times 50 \mu \mathrm{g} / \mathrm{Tag})$ kam es zu einer innerhalb der ersten Therapiewoche einsetzenden Verbesserung der Peak-flow-Werte der Patienten, parallel dazu ging der Bedarf an kurz wirksamen $\beta_{2}$-Sympathomimetika zurück. Die verbesserte Symptomkontrolle war während des gesamten Prüfzeitraumes von maximal 2,5 Jahren unverändert stabil. Nebenwirkungen auch der Langzeittherapie waren in erster Linie die pharmakologisch $\mathrm{zu}$ erwartenden unerwünschten Effekte von $\beta_{2}$-Sympathomimetika.

\section{Wirksamkeit}

Die Resultate der vorliegenden Studie ergänzen Ergebnisse großer kontrollierter Studien, die die bronchodilatatorische Wirkung der lang wirksamen $\beta_{2}$-Sympathomimetika Salmeterol und Formoterol gegenüber Plazebo und verschiedenen Vergleichsmedikamenten über Behandlungszeiträume von mehreren Wochen bis zu 12 Monaten bestätigen $[2,4,5,7$, 8,14,17-18]. Erstmalig werden die anhaltende Verbesserung der pulmonalen Funktion und der Rückgang des zusätzlich zur Basistherapie erforderlichen Bedarfs an kurz wirksamen $\beta_{2}$-Sympathomimetika über eine Therapiedauer von mehr als 2 Jahren belegt.

In die klinische Prüfung wurden Patienten mit leichter bis mittelschwerer obstruktiver Atemwegserkrankung eingeschlossen $[19,20]$. Das rekrutierte Patientenkollektiv ist daher repräsentativ für mehr als $90 \%$ der Patienten in der täglichen ärztlichen Praxis [21]. Bei der Auswahl der Teilnehmer wurde auf eine strenge Trennung zwischen Patienten mit Asthma bronchiale und chronisch-obstruktiver Bronchitis verzichtet, da diese Differenzierung im klinischen Alltag mit realistischem Aufwand häufig nicht durchführbar ist. Die möglicherweise unzureichende Klassifizierung der Patienten könnte andererseits die für ein Kollektiv von Patienten mit obstruktiven Atemwegserkrankungen niedrige Inzidenz atopischer Begleiterkrankungen und die überraschende Feststellung erklären, dass die Verbesserung der Lungenfunktion durch Therapie mit Salmeterol eher unabhängig von der Grunderkrankung war. Diese Einschränkung betrifft jedoch nicht die aus dieser Studie abzuleitende Schlussfolgerung, dass auch unter den Bedingungen der täglichen ärztlichen Praxis die Therapie mit Salmeterol langfristig gut wirksam ist.

Die festgestellte Steigerung der Peak-flow-Werte ist mit 46 l/ min realistisch, klinisch relevant und liegt etwas über den Ergebnissen einer ähnlich angelegten, kontrollierten Doppelblindstudie [15], in der unter Salmeteroltherapie $(2 \times 50 \mu \mathrm{g} /$ Tag) die Peak-flow-Werte innerhalb der ersten Woche um $27 \mathrm{l} / \mathrm{min}$ morgens und um $16 \mathrm{l} / \mathrm{min}$ abends anstiegen, während unter Therapie mit Salbutamol $(4 \times 400 \mu \mathrm{g} / \mathrm{Tag})$ lediglich Anstiege um 3 1/min bzw. 8 1/min zu verzeichnen waren. Auch der festgestellte Rückgang des zusätzlichen Verbrauchs kurz wirksamer $\beta_{2}$-Sympathomimetika ist quantitativ und qualitativ mit den Ergebnissen kontrollierter Prüfungen mit Salmeterol und Formoterol vergleichbar $[15,22]$. Diese Befunde 
belegen zudem, dass die Reduktion des Verbrauches kurz wirksamer $\beta_{2}$-Sympathomimetika unter Therapie mit lang wirksamen $\beta_{2}$-Sympathomimetika nicht bloß ein „virtueller“ Effekt ist, d.h. der Ersatz eines $\beta_{2}$-Sympathomimetikums durch ein anderes, sondern eine echte Einsparung und therapeutischen Zugewinn bedeutet.

Unter einer regelmäßigen Therapie mit lang wirksamen $\beta_{2^{-}}$ Sympathomimetika kann es zu einer Toleranzentwicklung zumindest im Hinblick auf die bronchoprotektive Wirkung kommen [11 -13]. Ursächlich könnten sowohl eine Abnahme der Wirkdauer bei erhaltener Wirkstärke [12], eine insgesamt verringerte Wirkstärke [12,23], als auch Änderungen in der Rezeptorkinetik unter Langzeittherapie sein [24]. Hierzu erlaubt die vorliegende Studie keine Aussage, da die bronchiale Hyperreagibilität nicht erfasst wurde. Es war bei Studienbeginn noch unklar, ob es unter einer regelmäßigen Therapie mit lang wirksamen $\beta_{2}$-Sympathomimetika auch zu einer Toleranzentwicklung der bronchodilatatorischen Wirkung kommt [3]. Obwohl in der vorliegenden Studie eine bronchodilatatorische Wirkung de facto nicht erfasst wurde (es wurden keine Reversibilitätsprüfungen im Verlauf durchgeführt), belegt die anhaltende Verbesserung der Lungenfunktion klar, dass mögliche Toleranzphänomene auch unter jahrelanger Therapie mit lang wirksamen $\beta_{2}$-Sympathomimetika für die Mehrzahl der Patienten ohne klinische Relevanz sind. Die Peak-flow-Werte der Patienten und der $\beta_{2}$-Mimetika-Verbrauch zeigten keinen Hinweis auf eine verminderte Wirksamkeit von Salmeterol mit zunehmender Anwendungsdauer als Hinweis auf eine Toleranzentwicklung. Vielmehr hatten die 345 Patienten, die über den gesamten Beobachtungszeitraum von 30 Monaten eine Salmeterol-Therapie durchgeführt hatten, stabile, bzw. noch im 3. Behandlungsjahr weiterhin leicht ansteigende Peak-flow-Werte. Diese Befunde werden zusätzlich durch die Resultate der Life-TableAnalyse gestützt. Die erzielten Verbesserungen der Peak-flowWerte blieben im Verlauf stabil, erneute Verschlechterungen traten nicht auf. Dennoch sollte die Möglichkeit einer Toleranzentwicklung bei einer Langzeittherapie mit lang wirksamen $\beta_{2}$-Sympathomimetika bedacht und bei entsprechendem Verdacht geprüft werden.

\section{Verträglichkeit}

Die vorliegende Studie bestätigt die gute Verträglichkeit von Salmeterol auch in der Langzeittherapie obstruktiver Atemwegserkrankungen über mehrere Jahre. Bislang liegen keine anderen prospektiven Studien vor, die diese Aussage $\mathrm{zu}$ Salmeterol erlauben. In dem in dieser Studie beobachteten Patientenkollektiv waren Exazerbationen der Grunderkrankung die häufigsten unerwünschten Ereignisse (58 Patienten, $9 \%$ ), die bei 13 Personen (2\%) zum Abbruch der Studie führten. Qualitativ und quantitativ ähnliche Ergebnisse wurden auch von anderen Untersuchern berichtet. Die Häufigkeit derartiger Exazerbationen wurde in anderen Langzeitstudien mit 10-15\% der Patienten pro Jahr unter Therapie mit Salmeterol $[15,25]$ und mit $22 \%$ der Patienten pro Jahr unter Therapie mit Plazebo angegeben [25]. Für die Therapie mit $\beta_{2^{-}}$ Sympathomimetika typische Nebenwirkungen (Tremor, Tachykardie, Herzklopfen und Muskelkrämpfe) wurden etwa gleich häufig wie in anderen Studien $[15,25]$ dokumentiert, wo derartige Ereignisse bei weniger als 5\% der Anwender aufgetreten waren. Eine speziell unter dem Gesichtspunkt der
Arzneimittelsicherheit angelegte Studie [25] zeigte dabei zwischen drei Behandlungsgruppen (Plazebo, Salmeterol und Salbutamol) keinen signifikanten Unterschied in der Häufigkeit unerwünschter Ereignisse bei 556 Patienten über einen Behandlungszeitraum von einem Jahr.

Die im Rahmen der vorliegenden Studie aufgetretenen Todesfälle waren vom Prüfarzt in keinem Fall mit der Salmeteroltherapie in Verbindung gebracht worden. Ein Todesfall war durch eine Exazerbation des zugrunde liegenden Asthma bronchiale bedingt. Dies mag für das untersuchte Kollektiv von Patienten mit leichter bis mittelschwerer Erkrankung zunächst ungewöhnlich erscheinen, kann aber bei der großen Anzahl untersuchter Patienten letztlich nicht ausgeschlossen werden.

Die vorgestellte Studie weist die üblichen prinzipiellen Schwächen offener, multizentrischer Medikamentenprüfungen auf. Zweifelsohne kann die alleinige Bestimmung des Peak flow, insbesondere bei Patienten mit einer COPD, nur sehr eingeschränkt als ausreichend repräsentativ für eine Verbesserung der Zielgröße „Lungenfunktion“ angesehen werden. $\mathrm{Zu}$ bemängeln ist ebenfalls die Auswahl der in die Prüfung eingeschlossenen Patienten, die teilweise obstruktive Atemwegserkrankungen nur leichter Ausprägung aufwiesen. Für dieses Patientenkollektiv sehen aktuelle Therapieempfehlungen eine Therapie mit einem lang wirksamen $\beta_{2}$-Sympathomimetikum nicht routinemäßig vor $[1,19,26]$. Ebenso konnten keine Informationen über Wirksamkeit und Verträglichkeit von Salmeterol bei Patienten mit schweren obstruktiven Atemwegserkrankungen gewonnen werden. Gerade diese Patienten stellen im klinischen Alltag jedoch häufig Problemfälle dar, deren Symptome schwer kontrollierbar sind und die zudem häufig eine relevante Ko-Morbidität aufweisen. Zu diesen Fragen sind zweifellos weitere Untersuchungen dringend wünschenswert.

Andererseits konnte durch das gewählte Studiendesign in kurzer Zeit eine große Zahl von Patienten und Prüfzentren rekrutiert werden. Die Studie fand unter realistischen Alltagsbedingungen statt, so dass ein repräsentatives Patientenkollektiv mit einer breiten Altersverteilung vorlag. Darüber hinaus wurden auch Patienten mit relevanter Ko-Morbidität in die Prüfung aufgenommen.

Aus diesen Befunden lässt sich schließen, dass das lang wirksame inhalative $\beta_{2}$-Sympathomimetikum Salmeterol bei Patienten mit leichten und mittelschweren obstruktiven Atemwegserkrankungen zu einer anhaltenden Verbesserung des Peak flows beiträgt und den Verbrauch an bedarfsadaptierten, kurz wirksamen $\beta_{2}$-Sympathomimetika zur Symptomkontrolle dauerhaft reduziert. Diese günstigen Effekte sind auch langfristig über eine Therapiedauer von $>2$ Jahren stabil, es fanden sich keine Hinweise auf klinisch relevante Toleranzphänomene. Die Verträglichkeit von Salmeterol ist auch in der Langzeitanwendung gut, das Spektrum der beobachteten Nebenwirkungen entspricht dem pharmakologischen Profil der Substanzklasse. Lang wirksame $\beta_{2}$-Sympathomimetika sind bei Patienten mit obstruktiven Atemwegserkrankungen auch in der Langzeittherapie unter Praxisbedingungen eine wirksame und gut verträgliche Erweiterung der therapeutischen Möglichkeiten. 


\section{Danksagung}

Die Autoren danken allen Prüfzentren der SAL-MULTI-Studiengruppe für die Teilnahme an der klinischen Prüfung. Die statistische Aufarbeitung erfolgte durch das Institut für angewandte Statistik GmbH, Bielefeld.

\section{Literatur}

${ }^{1}$ National Heart, Lung, and Blood Institute. Guidelines for the diagnosis and management of asthma. NIH Publication 1998; $98-4051$

${ }^{2}$ Greening AP, Ind PW, Northfield M, Shaw G. Added salmeterol versus higher-dose corticosteroid in asthma patients with symptoms on existing inhaled corticosteroid. Lancet 1994; 344: 219 224

${ }^{3}$ Pauwels RA, Löfdahl C, Postma DS, Tattersfield AE, O'Byrne P, Barnes PJ, Ullman A. Effect of inhaled Formoterol and Budesonide on exacerbations of asthma. N Engl J Med 1997; 337: $1405-1411$

${ }^{4}$ Woolcock A, Lundback B, Ringdal N, Jacques LA. Comparison of addition of salmeterol to inhaled steroids with doubling of the dose of inhaled steroids. Am J Respir Crit Care Med 1996; 153 : $1481-1488$

${ }^{5}$ Kesten S, Chapman KR, Broder I, Cartier A, Hyland RH, Knight A, Malo JL, Mazza JA, Moote DW, Small P, Tarlo S, Gontovnick L, Rebuck AS. A three-month comparison of twice daily inhaled formoterol versus four times daily inhaled albuterol in the management of stable asthma. Am Rev Respir Dis 1991; 144: $622-625$

${ }^{6}$ Pearlman DS, Chervinsky P, LaForce C, Seltzer JM, Southern DL, Kemp JP, Dockhorn RJ, Grossman J, Liddle RF, Yancey SW, Cocchetto DM, Alexander WJ, van As A. A comparison of salmeterol with albuterol in the treatment of mild-to-moderate asthma. N Engl J Med 1992; 327: 1420 - 1425

${ }^{7}$ Pearlman DS, Liddle R. Controlling asthma symptoms; salmeterol compared with salbutamol in large-scale multi-centre studies. Eur Respir Rev 1994; 21: 301 - 305

${ }^{8}$ Cazzola M, Matera MG, Santangelo G, D'Amato G. Salmeterol and formoterol in partially reversible severe chronic obstructive pulmonary disease: a dose-response study. Respir Med 1995; 89: $357-362$

${ }^{9}$ Grove A, Lipworth BJ, Reid P, Smith RP, Ramage L, Ingram CG, Jenkins RJ, Winter JH, Dhillon DP. Effects of regular salmeterol on lung function and exercise capacity in patients with chronic obstructive airways disease. Thorax 1996; 51: 689 - 693

${ }^{10}$ Ulrik CS. Efficacy of inhaled salmeterol in the management of smokers with chronic obstructive pulmonary disease: a single centre randomised, double blind, placebo controlled, crossover study. Thorax 1995; 50: 750 - 754

${ }^{11}$ Drotar DE, Davis EE, Cockcroft DW. Tolerance to the bronchoprotective effect of salmeterol 12 hours after starting twice daily treatment. Annals of Allergy, Asthma, \& Immunology 1998; 80: $31-34$

12 Nelson JA, Strauss L, Skowronski M, Ciufo R, Novak R, McFadden ER. Effect of long-term salmeterol treatment on exercise-induced asthma. N Engl J Med 1998; 339: 141 - 146

${ }^{13}$ Ramage L, Lipworth BJ, Ingram CG, Cree IA, Dhillon DP. Reduced protection against exercise induced bronchoconstriction after chronic dosing with salmeterol. Respir Med 1994; 88: 363 - 368

${ }^{14}$ Britton MG, Earnshaw JS, Palmer JB. A twelve month comparison of salmeterol with salbutamol in asthmatic patients. Eur Respir J 1992; 5: $1062-1067$

${ }^{15}$ Lundback B, Rawlinson DW, Palmer JB. Twelve month comparison of salmeterol and salbutamol as dry powder formulations in asthmatic patients. Thorax 1993; 48: 148 - 153
${ }^{16}$ Quanjer PH, Tammeling GJ, Cotes JE, Pedersen OF, Peslin R, Yernault JC. Lung volumes and forced ventilatory flows-Standardization of lung function tests. Eur Respir J 1993; 6 (Suppl. 16): $5-40$

${ }^{17}$ D'Alonzo GE. Efficacy of inhaled salmeterol in the treatment of asthma. Eur Respir Rev 1995; 27: 128 - 132

${ }^{18}$ Faurschou P, Steffensen I, Jacques L. Effect of addition of inhaled salmeterol to the treatment of moderate-to-serve asthmatic uncontrolled on high-dose inhaled steroids. Eur Respir J 1996; 9: $1885-1890$

${ }^{19}$ Wettengel R, Berdel D, Krause J, Kroegel C, Kroidl RF, Leupold W, Lindemann H, Magnussen H, Meister R, Morr H, Nolte D, Rabe K, Reinhardt D, Sauer R, Schultze-Werninghaus G, Ukena D, Worth $\mathrm{H}$. Empfehlungen zur Asthma-Therapie bei Kindern und Erwachsenen. Pneumologie 1999; 52: 591 - 601

${ }^{20}$ Wettengel R, Böhning W, Cegla U, Criée C, Fichter J, Geisler L, Fabel H, Köhler D, Konietzko N, Lindemann H, Magnussen $H$, Matthys H, Meister R, Morr H, Nolte D, Petro W, SchultzeWerninghaus G, Sill V, Sybrecht G, Wiesner B, Worth H. Empfehlungen der Deutschen Atemwegsliga zur Behandlung von Patienten mit chronisch obstruktiver Bronchitis und Lungenemphysem. Med Klin 1995; 90: 3 - 7

${ }^{21}$ Magnussen H. Asthma bronchiale. Pneumologie 1996; 50: 578 581

${ }^{22}$ Häcki MA, Hinz GW, Medici TC. Clinical experience over five years of daily therapy with formoterol in patients with bronchial asthma. Clin Drug Invest 1997; 14: 165 - 174

${ }^{23}$ Tan S, Hall IP, Dewar J, Dow E, Lipworth B. Association between Beta-adrenoceptor polymorphism and susceptibility to bronchodilator desensitisation in moderately severe stable asthmatics. Lancet 1997; 350: 995 - 999

${ }^{24}$ January B, Seibold A, Allal C, Whaley BS, Knoll BJ, Moore RH, Dickey BF, Barber R, Clark RB. Salmeterol-induced desensitization, internalization and phosphorylation of the human beta2adrenoceptor. Br J Pharmacol 1998; 123: 701 - 711

${ }^{25}$ Nathan RA, Seltzer JM, Kemp JP, Chervinsky P, Alexander JW, Liddle R, Mills R. Safety of salmeterol in the maintenance therapy of asthma. Ann Allergy Asthma Immunol 1995; 75: $243-248$

${ }^{26}$ AAAAI Committee on drugs. Safety and appropriate use of salmeterol in the treatment of asthma. J Allergy Clin Immunol 1996; 98 : 475 - 480

Dr. med. K.-M. Beeh

Schwerpunkt Pneumologie

III. Medizinische Klinik und Poliklinik

55131 Mainz

E-mail: k.beeh@3-med.klinik.uni-mainz.de 